\title{
Family Systems Perspective and Diabetes Type I: Reflective Analysis
}

\author{
Charity Perry \\ Department of Sociology, California State University, Los Angeles, USA \\ Email: cperry5@calstatela.edu
}

Received 16 July 2015; accepted 1 November 2015; published 4 November 2015

Copyright (C) 2015 by author and Scientific Research Publishing Inc.

This work is licensed under the Creative Commons Attribution International License (CC BY). http://creativecommons.org/licenses/by/4.0/

c) (i) Open Access

\begin{abstract}
The purpose of this paper is to reflectively explore diabetes from a family systems perspective. Specific discussion will involve current understanding of Type I Diabetes and Family Systems, understanding suffering of illness experience, and will provide comparison and contrast of effective behavioral interventions for families under the care of a marriage and family therapist. While moving expectations toward systemic understanding of Type I Diabetes will be explored, and a brief account of the researchers' personal and present familial history within the discipline of marriage and family therapy will also be discussed.
\end{abstract}

\section{Keywords}

Family Systems, Type I Diabetes, Marriage and Family Therapy, Chronic Illness, Behavioral Interventions

\section{Introduction}

Historically, Diabetes Type I has been defined as a chronic illness. Diabetes is a disease in which the body does not produce or properly use insulin to convert starches, sugar and other foods into energy which is needed for daily life. There are two major types of diabetes. Type I Diabetes is an autoimmune disease in which the body does not produce insulin. Type I Diabetes often occurs in childhood; however, late onset Type I Diabetes occurs; Type I diabetics must inject insulin daily to maintain life. Type II Diabetes is a metabolic disorder in which the body cannot produce enough insulin, and is the most common form of diabetes, and is reaching epidemic proportions. Signs of either type cannot always be recognized; for proper diagnosis lab tests are required. The purpose of this reflective analysis is to present a brief account of the researcher's personal and present familial history with Diabetes (Type I and II) while providing comparison and contrast of effective behavioral interventions for families under the care of a marriage and family therapist and exploring the topic through a family systems perspective. 


\section{Current Understanding of Type I Diabetes and Family Systems}

A central premise of family systems theory is how family systems organize themselves to carry out the daily challenges and tasks of life. Adjusting to the developmental needs of its members is also critical to carrying out the tasks. A family systems perspective argues in order to understand a family system we must look at the family as a whole.

According to Shrivastava, Shrivastava and Ramasamy (2013) “diabetes mellitus (DM) is a chronic progressive metabolic disorder characterized by hyperglycemia mainly due to absolute (Type 1 DM) or relative (Type 2 DM) deficiency of insulin hormone.” (p. 14) The affects of DM can be seen on virtually every system of the body due to metabolic disturbances caused by hyperglycemia. This is particularly evident if there is suboptimal control over diabetes during one's lifetime. According to World Health Organization (WHO) more than 346 million people are estimated to have DM.

Research is limited on diabetes-related family conflict and tends to be a prime research starting point for families who seek out a greater understanding of a family member with the disease, and what family support is needed to aid in the assistance of Type I Diabetes. Considering that Type I Diabetes is a chronic illness, it may be managed by an individual, their family, and the healthcare system. Due to familial interactions, family communication is essential for the benefit of the individual and homeostasis of the family. McDaniel, Hepworth and Doherty (1992) developed a general approach for marriage and family therapists in an effort to strategize theoretical and clinical contributions applicable to chronic illnesses. Caregivers (part-time or full-time) can initiate therapy for themselves. As familial demands increase, the overwhelming feelings of assisting those with chronic illness can increase. During such cases, as these authors recognize, family members can be included in caregiver's therapy when necessary.

Steinglass and Horan (1988) identified models within a family which acts as platform to recognize if a family aligns to a resource or deficit model. In families who adhere to positive family functions such as social support are buffers for the illness severity. Families with attributions of characteristics such as enmeshment, rigidity, or poor family conflict resolutions are said to impede optimal care and are described as a deficit model. Using systems theory and techniques, marriage and family therapists can assist in a family's natural response to various losses among the journey of a chronic illness. Such loss can be mitigated in a positive manner by a therapist as evidence of therapeutic change within a family system. Psychosocially, insulin-dependent, adult-onset diabetes (Type I) is a gradual, progressive, chronic illness which can shorten life span, and possibly be fatal.

\section{Understanding Suffering of Illness Experience}

Considering the life cycle of a chronic illness, it is essential to understand behavioral patterns of families who manage members with a chronic illness. Patterns of behavior are present in every family system, and may take the form of a spouse who may be overprotective and/or those spouses who present with a tolerance level for ambiguity. Under the general assumption flexibility is either/and, or both in their behavioral patterns, suggest a willingness to communicate which promotes healthy discussion among family members of their needs and desires.

Behavioral family systems therapy for diabetes (Wysocki et al., 2007) was examined to understand the maintenance of effects within a family where a member was a diabetic. This research focused on diabetes-related family conflict, treatment adherence, and metabolic control. For this study it should be noted families meeting criteria for the study such as: absence of severe psychopathology or substance abuse, English literate, geographical stability and established care for Type I diabetes were sought. This study also used adolescents within the study groups, but certainly the outcomes and application of the study can be used to understand suffering of illness experience in late onset Type I diabetes. The researchers concluded significant improvements in individual communications among family members existed along with an improvement of A1C levels in diabetics which refers to the average of plasma glucose concentration over long periods of time.

\section{Expectations of Chronic Illness and Moving Toward Systemic Understanding of Type I Diabetes}

Assumptions play a clear role in chronic illness, one can assume beneath the labeling of chronic illness such as Type I diabetes, a pre-existing notion of how the disease was diagnosed, maintained and its effects upon the family can be categorically recognized by assumption recognition. The first assumption we will make is diabetes is 
caused by lifestyle choices. This could be a common case for a Type II diabetic, which is associated with a food choice, lack of exercise routines and other factors. However, lifestyle choices do not specifically play a role in Type I Diabetes which accounts only for $5 \%-10 \%$ of all diabetic cases.

The second assumption is more dangerous than Type II diabetes. Under this general assumption, individuals may not take their blood sugar levels seriously, which can certainly have a domino effect on their body systems and take a toll on their family from a systemic perspective. When diabetes management is not seen as important by the individual, and is dismissed as important by the family, the entire family unit may suffer. Historically, these attitudes have led to death due to lack of insulin in Type I Diabetes because of the inability to diagnose and supply insulin to an insulin-dependent individual. For a Type II Diabetic, neuropathy, eye damage, or kidney failure may imply more danger over the life span that Type I Diabetes. If an individual does not control their diabetes the latency of Type II diabetes could in fact be deadly, just as a lack of insulin for the Type I Diabetic.

Both assumptions can be held to specific standards medically, as insulin for the Type I diabetic is a life-saving therapy; however, the misuse or incorrect monitoring of insulin could be detrimental for the Type I diabetic. All of the assumptions discussed can potentially lead to creating a solid family therapy model for diabetics and their families, encourage support for the individual with the chronic illness by giving families time to consider the chronic illness at hand, and coping mechanisms which will increase family unity rather than family members withdrawing emotionally and physically to cope

\section{Effective Behavioral Interventions for Families under the Car of a Marriage and Family Therapist: Comparison and Contrast}

Outlining the similarities and differences in behavioral interventions includes components of treatment plans such as the use of approaches grounded in theory compared with approaches not grounded in theory (Hood \& Nansel, 2007). Some marriage and family therapists may not use theory to form any sort of perspective with a family being counseled initially, but it does seem as though having some framework is helpful in assessing the many conflicts which can arise. To determine the best approach as a marriage and family therapist, especially when providing therapy for those with a chronic illness would be a consideration of the therapists family medical history (past or present) along with being mindful of the biases toward the illness, or how a therapist may have experienced chronic illness within his/her family system.

\section{Other Similarities and Differences among Therapists}

Some therapists may employ the used of a diabetes-specific intervention. Discussed previously was the idea of flexibility. Flexibility seems to be a key in similarities of a successful family unit who is faced with the challenges of a chronic illness, along with communication about needs individually. This research also discusses sustainability, regardless of the model to be effective for the individual, and family as a unit. Application or development of approaches by a family therapist and the utilization and effectiveness of such an individual to assist families is important to future research, especially consideration of those with Type I (adult onset) diabetes.

\section{Researcher's Lived Experience}

The researcher's family members are taken as examples to illustrate family therapy of Type I Diabetes. The purpose of taking the researcher's grandmother and husband in this body of research is to identify with readers who may have similar life experiences. Even though family units may perceive their reality differently, one can find comfort in reading other life experiences in which they are not alone in their plights (former, present, or future).

Individualistic suffering and relational suffering can be dichotomous, because of the way a family unit perceives its reality. Exploring the process has been a significant part of my growth as a spouse to an individual with the chronic illness, Type I Diabetes. As a partner, many roles are embraced and must be acted upon in whatever capacity a situation may call for. My grandmother, who was my first recollection of a diabetic (Type II) in our family lost her eyesight, ultimately being able to only see shadows but carried on for years as though she could see, thus withholding from the family as she was a caretaker of her husband who was failing in his own health. An example of my personal experience was finding the cellophane from meat she had cooked, along with the meal she was preparing, thus prompting me to question my father about what had taken place. It became more apparent to many 
in the family the signs were there, but it was not always obvious to the family she was nearly blind. Once it was established her eyesight was failing, if and when she needed to use insulin, she could not see the gage to know the amount was accurate, essentially she would guess. She may have asked us to see what numbers were there, but honestly if that happened I do not recall. The researcher's family members are taken as examples to illustrate family therapy of Type I Diabetes. What is the purpose of taking the researcher's grandmother as an example? This case needs further analysis.

My husband is a Type I diabetic. By all measures we are a highly functional family while faced with a chronic illness. As partners raising four children, we make adjustments to daily schedules if we need to (an example of a frequent adjustment to our daily schedule is if my husband has experienced significant highs or lows in his blood sugar during the night, and he had initially planned to take the children to school the following morning), I will take them instead. Thanks to awareness and his communication to me about the event); another adjustment is cooking meals that have less processed foods (when we cook dinner for the family); and making sure he knows in advance what time meals are occurring on date nights and in general so he can plan his food intake for the day. These seem minor to us, and through open communication, the familial needs are met, along with all of our individual needs as well.

\section{Conclusion}

By gaining insight into Type I and II diabetes and through the reflection of the authors lived experience, it is important reminder that we must advance the knowledge of collaborative healthcare models from a family systems perspective and therapy. By creating a greater understanding of this topic, early involvement of patients and their families may create the best foundation for those who are recently diagnosed with Type I or II diabetes. While the stories of individual families carry unique components, there are often common themes, across families with members who have chronic illness. Once a therapist has elicited the family's story, stress patterns within the family system can be addressed, ultimately setting the family on course to halt patterns which may further perpetuate stress due to a chronic illness. "Effective diabetes management not only requires the provision of comprehensive medical care and diabetes education; skillful self care by the person with diabetes is essential. A person's self-care practices are influenced by their diabetes knowledge, their physical ability to undertake tasks such as blood glucose testing, the support they receive from family and health professionals, and their own beliefs and attitudes. A person's beliefs and attitudes develop throughout life as a result of upbringing, culture and life experiences.” (Dunning, 2004: p. 30)

\section{Future Research}

There is some limited research on family conflict and adolescents; however, there is a complete deficit of research examining family conflict when exploring the topic of late-onset Type I diabetes and the effects upon a family unit. Efforts in research should move to understanding, guiding, and therapeutic interventions for families with lateonset diabetes who may experience conflict due to such diagnoses.

\section{References}

Dunning, T. (2004). Exploring the World Mythology of Diabetes. Diabetes Voice, 49, 30-33.

Hood, K., \& Nansel, T. (2007). Commonalities in Effective Behavioral Interventions for Children and Adolescents with Type 1 Diabetes: A Review of Reviews. Diabetes Spectrum, 20, 251-254. http://dx.doi.org/10.2337/diaspect.20.4.251

http://diabetes.about.com/od/familymatters/a/adultstype1.htm

http://www.pamf.org/teen/health/diseases/diabetes.html

McDaniel, S., Hepworth, J., \& Doherty, W. (1992). Medical Family Therapy: A Biopsychosocial Approach to Families with Health Problems. New York: Basic Books.

Shrivastava, S. R., Shrivastava, P. S., \& Ramasamy, J. (2013). Role of Self-Care in Management of Diabetes Mellitus. Journal of Diabetes \& Metabolic Disorders, 12, 14. http://dx.doi.org/10.1186/2251-6581-12-14

Steinglass, P., \& Horan, M. (1988). Families and Chronic Medical Illness. Journal of Psychotherapy \& the Family, 3, 127142. http://dx.doi.org/10.1300/J287v03n03_09

Wysocki, T., Harris, M., Buckloh, L., Mertlich, D., Lochrie, A., Mauras, N., \& White, N. (2007). Randomized Trial of Behavioral Family Systems Therapy for Diabetes. Diabetes Care, 30, 555-560. http://dx.doi.org/10.2337/dc06-1613 